\title{
Índices de competição em árvores individuais de eucalipto
}

\author{
Fabrina Bolzan Martins ${ }^{(1)}$, Carlos Pedro Boechat Soares ${ }^{(2)}$, Helio Garcia Leite(2), \\ Agostinho Lopes de Souza(2) e Renato Vinícius Oliveira Castro(2)
}

\begin{abstract}
(1)Universidade Federal de Itajubá, Instituto de Recursos Naturais, CEP 37500-903 Itajubá, MG. E-mail: fabrinabm@gmail.com (2)Universidade Federal de Viçosa, Departamento de Engenharia Florestal, CEP 36570-000 Viçosa, MG. E-mail: csoares@ufv.br, hgleite@gmail.com, alsouza@ufv.br, castrorvo@ymail.com
\end{abstract}

\begin{abstract}
Resumo - O objetivo deste trabalho foi avaliar cinco índices de competição independentes da distância, em árvores individuais de plantios comerciais de eucalipto, e verificar a possibilidade de ajuste de uma equação única para o crescimento em diâmetro e altura, bem como a probabilidade de mortalidade para três classes de produtividade. Foram avaliados dados de 30 parcelas permanentes de plantios clonais híbridos, não desbastados, de Eucalyptus grandis $\mathrm{x}$ Eucalyptus urophylla. Os índices de competição foram avaliados por meio do coeficiente de correlação simples e o teste F-parcial, juntamente com o teste de identidade, para verificar a possibilidade de ajuste de uma equação única para três classes de produtividade. $\mathrm{O}$ índice de área basal foi o que apresentou o melhor desempenho. Ao contrário do observado quanto ao crescimento em diâmetro e altura, para a probabilidade de mortalidade, foi possível o ajuste de uma única equação para as três classes de produtividade.
\end{abstract}

Termos para indexação: Eucalyptus, floresta equiânea, índices independentes da distância, modelo de crescimento para árvore individual.

\section{Competition indexes for individual eucalyptus trees}

\begin{abstract}
The objective of this work was to evaluate five distance-independent competition indexes, in individual trees of an eucalyptus commercial plantation, and to verify the possibility of fitting a single equation for growth and height, as well as the probability of mortality for three yield classes. Data from 30 permanent plots of unthinned clonal hybrids of a Eucalyptus grandis x Eucalyptus urophylla forest, were analyzed. The competition indexes were evaluated through the simple correlation coefficient and the partial-F test, together with an identity test to verify if a single equation could be adjusted for the three yield classes. The basal area index performed better than other competition indexes. Contrarily to what was found for diameter and height, it was possible to adjust a single equation for the three yield classes for the probability of mortality.
\end{abstract}

Index terms: Eucalyptus, even-aged forests, distance-independent indexes, individual tree growth model.

\section{Introdução}

A competição entre plantas inicia-se quando os recursos necessários para seu crescimento decrescem abaixo de sua demanda, afetando o crescimento das plantas (Weber et al., 2008).

A competição é uma variável quantitativa importante em modelos de crescimento e produção florestal. No entanto, é difícil de ser mensurada, pois não se conhecem suas causas diretas, bem como a ligação entre a competição, a redução dos recursos disponíveis e a redução da taxa de crescimento. $\mathrm{Na}$ área florestal, a competição pode ser estimada por intermédio dos índices de competição, que permitem quantificar o nível competitivo de uma árvore (árvore-objeto) em relação ao de suas competidoras (Davis et al., 2005).
Numerosos índices de competição têm sido desenvolvidos e aprimorados, para quantificar o status competitivo de árvores individuais em determinados povoamentos (Daniels, 1976; Sterba \& Monserud, 1997; Fox et al., 2007; Castagneri et al., 2008). Alguns expressam o estresse imposto pela competição, e outros representam a quantidade de recursos disponíveis para a árvore (Eid \& Tuhus, 2001). Esses índices são utilizados nos modelos para árvore individual (Zhao et al., 2006; Crecente-Campo et al., 2009; Chassot et al., 2011), para povoamento (Sánchez-González et al., 2006) e para classes de tamanho (Cao, 1997, 2006).

Os índices de competição são divididos em três categorias: independentes da distância, que utilizam variáveis em nível de povoamento e 
dimensões iniciais da árvore-objeto; dependentes da distância, que incorporam além das variáveis mencionadas anteriormente, o número, as dimensões e a localização das árvores vizinhas ou competidoras com a árvore-objeto (Tomé \& Burkhart, 1989); e semi-independentes da distância, similares aos índices independentes; no entanto, as semi-independentes são computadas, considerando-se parcelas circulares ao redor da árvore-objeto (Stage \& Ledermann, 2008).

Vários estudos têm sido realizados para comparar índices de competição independentes e dependentes da distância entre árvores. Teoricamente, os índices dependentes da distância deveriam apresentarvantagens sobre os independentes da distância; no entanto, não há confirmação da superioridade universal de um tipo de índice sobre o outro nem sobre qual é o melhor índice dentro de cada categoria (Daniels et al., 1986; Fox et al., 2007; Chassot et al., 2011). Além disso, a grande maioria desses índices foi avaliada em florestas nativas e temperadas (Cao, 1997; Zhang et al., 2004; Crecente-Campo et al., 2009) e são pouco estudados tanto em florestas plantadas quanto em florestas naturais no Brasil (Cunha, 2009; Chassot et al., 2011). Outra preocupação, ao se estudar e obter os índices de competição, diz respeito à necessidade ou não de se ajustar uma equação comum para o crescimento (diâmetro e altura) e mortalidade (probabilidade de mortalidade) para diferentes classes de produtividade.

O objetivo deste trabalho foi avaliar cinco índices de competição, independentes da distância, para plantios comerciais clonais de eucalipto. Verificou-se, ainda, a possibilidade de ajuste de uma equação única para o crescimento em diâmetro e altura, e a probabilidade de mortalidade para três classes de produtividade.

\section{Material e Métodos}

Este trabalho foi realizado com dados de inventários florestais contínuos (entre 1997 e 2003), de plantios clonais híbridos não desbastados de Eucalyptus grandis W. Hill ex Maiden x Eucalyptus urophylla S.T. Blake, provenientes da empresa Jari Celulose S.A., com sede

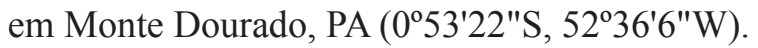

Segundo a classificação de Köppen, o clima da região é do tipo Am, com precipitação média anual de $2.115 \mathrm{~mm}$ e curta estação seca entre setembro e novembro. A temperatura média anual é de $26,4^{\circ} \mathrm{C}$, e a umidade relativa média oscila entre 80 e $85 \%$ (Demolinari, 2006; Martins, 2011).
Foram utilizados dados de 30 parcelas permanentes, de formato retangular com $500 \mathrm{~m}^{2}$ de área útil, e espaçamento entre plantas de $3 \times 3 \mathrm{~m}$ (Tabela 1). Em cada parcela, foram realizadas pelo menos cinco medições, tendo-se considerado as classes de produtividade alta, média e baixa.

Em cada parcela, foram mensurados os diâmetros, a $1,30 \mathrm{~m}$ do solo (DAP), maiores que 4,0 cm, as alturas total $(\mathrm{Ht})$ das 15 primeiras árvores e a altura total $(\mathrm{Hd})$ das cinco árvores dominantes. Para estimar a altura total das demais árvores da parcela, utilizou-se a equação hipsométrica, ajustada para a área de estudo (Demolinari, 2006):

$\mathrm{Ht}=36,9876-30,4340 \exp (-0,000499$ (DAP $\times$ $\operatorname{lnHd} \times \operatorname{lnI})^{1,388275}, \overline{\mathrm{R}}^{2}=83,7 \% ; \mathrm{S}_{\mathrm{yx}} \%= \pm 11,79 \%$,

em que: Hd é a altura média das árvores dominantes, em m; I é a idade, em meses; $\overline{\mathrm{R}}^{2}$ é o coeficiente de determinação ajustado, em percentagem e $\mathrm{S}_{\mathrm{yx}} \%$ é o erro-padrão da estimativa, em percentagem, ambos calculados na unidade original da variável dependente, em $\mathrm{m}$.

Para a classificação da capacidade produtiva, foi utilizada a equação ajustada por Demolinari (2006) para a mesma área de estudo, como:

$$
\ln \mathrm{S}=\ln H d+14,88011596\left(1 / \mathrm{I}_{\mathrm{i}}-1 / \mathrm{I}\right),
$$

em que: $\mathrm{S}$ é o índice de local; $\mathrm{I}_{\mathrm{i}}$ é a idade índice, 60 meses.

Para a classificação das parcelas, as seguintes classes de produtividade foram consideradas: baixa $(S=20)$, parcelas com $\overline{\mathrm{Hd}} \leq 23 \mathrm{~m}$ na idade índice de 60 meses; média $(\mathrm{S}=26)$, parcelas com $\overline{\mathrm{Hd}}$ entre 24 e $29 \mathrm{~m}$; alta $(\mathrm{S}=32)$, parcelas com $\overline{\mathrm{Hd}}>29 \mathrm{~m}$.

Foram avaliados e comparados os índices de competição independentes da distância (IID) (Martins, 2011):

$$
\begin{aligned}
& \mathrm{IID}_{1}=\mathrm{d}_{\mathrm{i}}^{2} / \overline{\mathrm{D}}^{2}, \mathrm{IID}_{2}=\mathrm{h}_{1} / \overline{\mathrm{H}}, \mathrm{IID}_{3}=\mathrm{d}\left(\mathrm{d}_{\mathrm{i}}^{2} \times \mathrm{hi}\right) /\left(\overline{\mathrm{D}}^{2} \times \overline{\mathrm{H}}\right), \\
& \mathrm{IID}_{4}=\mathrm{BAI}=\mathrm{d}_{\mathrm{i}}^{2} / \mathrm{q}^{2}, \mathrm{IID}_{5}=\mathrm{BAL}_{\mathrm{i}}=\sum_{\mathrm{i}=1}^{\mathrm{n}} \pi \mathrm{DAP}^{2} / 4,
\end{aligned}
$$

em que: $\mathrm{d}_{\mathrm{i}}$ é o diâmetro (DAP) da árvore-objeto, em cm; $\overline{\mathrm{D}}$ é a média aritmética dos diâmetros das árvores da unidade amostral, em $\mathrm{cm}$; $\mathrm{h}_{\mathrm{i}}$ é a altura total da árvore objeto, em $\mathrm{m} ; \overline{\mathrm{H}}$ é a altura média das árvores da unidade amostral, em m; q é o diâmetro quadrático da unidade amostral, em cm; BAI é o índice de área basal; e $\mathrm{BAL}_{\mathrm{i}}$ é o somatório das áreas seccionais das árvores maiores que a árvore-objeto. 
Inicialmente, verificou-se o comportamento dos índices de competição em função da idade, tendo-se analisado graficamente a tendência média de cada um dos índices em relação à idade, para cada classe de produtividade. Após esta etapa, realizou-se a análise de correlação simples entre os índices de competição e as variáveis de crescimento (diâmetro e altura), mortalidade e variáveis do povoamento (idade e área basal), para cada classe de produtividade, conforme sugerido por Daniels (1976), Daniels et al. (1986), Tomé \& Burkhart (1989) e Sánchez-González et al. (2006). Além da correlação simples, foi avaliada a significância estatística dos índices com maior correlação simples, na presença de outras variáveis explicativas, mediante o uso de regressões múltiplas baseadas nos modelos teóricos. Para isso, foi utilizado o teste F-parcial, dado pela expressão (Neter et al., 1996):

$\mathrm{F}\left(\mathrm{x}_{1} \mid \mathrm{x}_{2}, \mathrm{x}_{3} \ldots, \mathrm{x}_{\mathrm{n}}\right)=\mathrm{SQ}_{\mathrm{reg}}\left(\mathrm{x}_{1}, \mathrm{x}_{2}, \mathrm{x}_{3} \ldots, \mathrm{x}_{\mathrm{n}}\right)-\mathrm{SQ}_{\mathrm{reg}}\left(\mathrm{x}_{2}, \mathrm{x}_{3} \ldots\right.$, $\left.\mathrm{x}_{\mathrm{n}}\right) / \mathrm{QM}_{\mathrm{res}}\left(\mathrm{x}_{1}, \mathrm{x}_{2}, \mathrm{x}_{3} \ldots, \mathrm{x}_{\mathrm{n}}\right)$,

em que: $\mathrm{F}\left(\mathrm{x}_{1} \mid \mathrm{x}_{2}, \mathrm{x}_{3} \ldots, \mathrm{x}_{\mathrm{n}}\right)$ é o valor do teste F-parcial para a variável $\mathrm{X}_{1}$, na presença das demais variáveis; $\mathrm{SQ}_{\text {reg }}$ é a soma de quadrados da regressão do modelo completo; $\mathrm{SQ}_{\text {reg' }}$ é a soma de quadrados da regressão do modelo reduzido; e $\mathrm{QM}_{\mathrm{res}}$ é o quadrado médio do resíduo do modelo completo.

$\mathrm{O}$ valor de $\mathrm{F}$ calculado $\left(\mathrm{F}_{\text {cal }}\right)$ foi comparado ao valor de $\mathrm{F}$ tabelado $\left(\mathrm{F}_{\mathrm{tab}}\right)$, a $5 \%$ de probabilidade.

Para aplicar o teste F-parcial, com cada classe de produtividade, foram ajustadas as regressões múltiplas referentes aos modelos teóricos (Martin \& Ek, 1984; Zhang et al., 2004; Davis et al., 2005):

$\Delta \mathrm{h}=\mathrm{f}(\mathrm{IC}, \mathrm{DAP}, \mathrm{B}, \mathrm{I}), \Delta \mathrm{d}=\mathrm{f}(\mathrm{IC}, \mathrm{B}, \mathrm{I}), \mathrm{PM}=\mathrm{f}(\mathrm{IC}, \mathrm{DAP}, \mathrm{I})$,

em que: $\Delta$ h é o crescimento em altura, em m; $\Delta \mathrm{d}$ é o crescimento em diâmetro, em cm; PM é a probabilidade de mortalidade ou percentual de árvores mortas, em cada classe de DAP; IC é o índice de competição, B é a área basal do povoamento, em $\mathrm{m}^{2} \mathrm{ha}^{-1}$.

A PM foi obtida pela expressão, adaptada de Rossi et al. (2007):

$$
P M=N_{j(t-1)}-N_{j(t)} / \sum_{i=1}^{j} N_{j(t-1)}
$$

em que: $\mathrm{N}_{\mathrm{j}(\mathrm{t}-1)}$ é o número de árvores vivas, na j-ésima classe de diâmetro, no início do período; e $\mathrm{N}_{\mathrm{j}(t)}$ é o

Tabela 1. Diâmetro à altura do peito (DAP), altura total $(\mathrm{Ht})$ e área basal $(\mathrm{B})$, por idade e classes de produtividade ${ }^{(1)}$ de árvores individuais de Eucalyptus grandis x Eucalyptus urophylla.

\begin{tabular}{|c|c|c|c|c|c|c|c|c|c|}
\hline \multirow{2}{*}{$\begin{array}{l}\text { Idade } \\
\text { (meses) }\end{array}$} & \multicolumn{3}{|c|}{ Produtividade alta } & \multicolumn{3}{|c|}{ Produtividade média } & \multicolumn{3}{|c|}{ Produtividade baixa } \\
\hline & Mínimo & Médio & Máximo & Mínimo & Médio & Máximo & Mínimo & Médio & Máximo \\
\hline & \multicolumn{9}{|c|}{$\operatorname{DAP}(\mathrm{cm})$} \\
\hline 24 & 4,77 & 13,12 & 18,62 & 4,33 & 10,86 & 16,33 & 4,04 & 8,88 & 14,01 \\
\hline 36 & 4,93 & 14,71 & 22,28 & 4,77 & 12,51 & 19,99 & 4,33 & 9,95 & 17,70 \\
\hline 48 & 5,00 & 15,61 & 24,67 & 5,00 & 13,63 & 23,11 & 4,80 & 10,85 & 20,21 \\
\hline 60 & 5,60 & 16,45 & 27,12 & 5,57 & 14,59 & 24,92 & 4,90 & 11,54 & 22,60 \\
\hline 72 & 7,03 & 17,31 & 29,44 & 6,30 & 16,34 & 27,69 & 5,00 & 13,34 & 23,43 \\
\hline \multirow[t]{2}{*}{84} & $-(2)$ & - & - & 6,62 & 17,19 & 30,40 & 5,01 & 13,92 & 25,37 \\
\hline & \multicolumn{9}{|c|}{$\mathrm{Ht}(\mathrm{m})$} \\
\hline 24 & 9,50 & 16,90 & 21,70 & 9,00 & 14,40 & 19,3 & 8,50 & 12,40 & 16,80 \\
\hline 36 & 10,50 & 20,60 & 26,80 & 10,00 & 17,80 & 24,6 & 9,30 & 14,60 & 21,70 \\
\hline 48 & 11,10 & 23,10 & 30,30 & 11,00 & 20,50 & 28,9 & 10,30 & 16,80 & 25,40 \\
\hline 60 & 12,60 & 24,90 & 32,70 & 11,90 & 22,50 & 31,4 & 10,70 & 18,30 & 28,50 \\
\hline 72 & 14,50 & 26,40 & 34,10 & 13,70 & 25,20 & 33,1 & 11,40 & 21,10 & 30,00 \\
\hline \multirow[t]{2}{*}{84} & - & - & - & 14,60 & 26,60 & 34,6 & 11,70 & 22,30 & 31,80 \\
\hline & \multicolumn{9}{|c|}{$\mathrm{B}\left(\mathrm{m}^{2} \mathrm{ha}^{-1}\right)$} \\
\hline 24 & 12,90 & 14,34 & 15,48 & 6,28 & 9,58 & 11,84 & 4,72 & 6,61 & 8,22 \\
\hline 36 & 15,70 & 17,76 & 18,77 & 8,11 & 12,73 & 16,10 & 7,01 & 8,22 & 10,07 \\
\hline 48 & 17,49 & 19,46 & 21,17 & 9,81 & 15,00 & 19,28 & 8,21 & 9,71 & 11,16 \\
\hline 60 & 16,73 & 21,15 & 22,83 & 11,93 & 17,18 & 21,46 & 8,93 & 11,00 & 12,27 \\
\hline 72 & 18,34 & 22,93 & 25,42 & 17,14 & 21,19 & 25,12 & 11,74 & 12,69 & 13,47 \\
\hline 84 & - & - & - & 19,08 & 23,27 & 27,53 & 12,58 & 13,83 & 14,82 \\
\hline
\end{tabular}

${ }^{(1)}$ Classe de produtividade alta (índice de local, $\mathrm{S}=32$ ): altura média das árvores dominantes $(\overline{\mathrm{Hd}})>29 \mathrm{~m}$; classe de produtividade média $(\mathrm{S}=26)$ : $\overline{\mathrm{Hd}}$ entre 24 e $29 \mathrm{~m}$; classe de produtividade baixa $(\mathrm{S}=20): \overline{\mathrm{Hd}} \leq 23 \mathrm{~m} .{ }^{(2)}$ Valores não observados. 
número de árvores vivas, na j-ésima classe de diâmetro no fim do período.

No caso das equações para o crescimento em altura e probabilidade de mortalidade, quando se utilizou o índice de competição $\mathrm{IID}_{4}$ (BAI), o DAP não foi utilizado como variável explicativa, em virtude de problemas de multicolinearidade. Nas equações para o crescimento em diâmetro, altura e probabilidade de mortalidade, quando se utilizou o índice de competição $\mathrm{IID}_{5}$ (BAL), a variável B não foi utilizada como variável explicativa, já que as duas variáveis explicativas apresentam tendência comum entre si, o que dificulta a estimação precisa dos coeficientes (Gujarati, 2006).

Aplicou-se o teste de identidade de modelos, com o objetivo de verificar a possibilidade de ajuste de uma equação única para o crescimento e mortalidade independente da classe de produtividade. Nessas equações, foi utilizado apenas o índice de competição selecionado, com base nas análises anteriores.

Para a realização do teste de identidade, foram criadas variáveis "Dummy" (Regazzi, 2003), as quais assumiram valores binários, 0 e 1 , conforme a classe de produtividade, da seguinte forma: $\mathrm{D}_{\mathrm{i}}=1$, se a árvore estiver presente na classe de produtividade $i ; D_{i}=0$, se $\mathrm{a}$ árvore estiver ausente da classe de produtividade $\mathrm{i}$.

Dessa forma, foi possível expressar as equações individuais, ajustadas para as três classes de produtividade (n) que têm $\mathrm{R}^{\mathrm{n}}$ regressões lineares múltiplas (Schneider \& Tonini, 2003):

$$
\begin{aligned}
& R^{1}: \hat{Y}_{1}=b_{11} X_{1}+b_{12} X_{2}+\ldots b_{1 m} X_{m} \\
& R^{2}: \hat{Y}_{2}=b_{21} X_{1}+b_{22} X_{2}+\ldots b_{2 m} X_{m} \\
& R^{3}: \hat{Y}_{3}=b_{31} X_{1}+b_{32} X_{2}+\ldots b_{3 m} X_{m}
\end{aligned}
$$

Todas as equações individuais foram agrupadas, para permitir a definição do modelo completo $\left(\mathrm{M}_{\mathrm{c}}\right)$, representado por:

$\mathrm{M}_{\mathrm{c}}: \hat{\mathrm{Y}}=\mathrm{b}_{11} \mathrm{X}_{11}+\mathrm{b}_{12} \mathrm{X}_{12}+\ldots+\mathrm{b}_{1 \mathrm{~m}} \mathrm{X}_{1 \mathrm{~m}}+\ldots$ $+b_{22} X_{22}+\ldots b_{2 m} X_{2 m}+\ldots+b_{s m} X_{s m}$, em que: $X_{11}=D_{1} X_{1} ; X_{12}=D_{1} X_{2} ; X_{1 m}=D_{1} X_{m}$; $\mathrm{X}_{21}=\mathrm{D}_{2} \mathrm{X}_{1} ; \mathrm{X}_{22}=\mathrm{D}_{2} \mathrm{X}_{2} ; \mathrm{X}_{2 \mathrm{~m}}=\mathrm{D}_{2} \mathrm{X}_{\mathrm{m}} ; \mathrm{X}_{31}=\mathrm{D}_{3} \mathrm{X}_{1}$; $\mathrm{X}_{32}=\mathrm{D}_{3} \mathrm{X}_{2}$; e $\mathrm{X}_{3 \mathrm{~m}}=\mathrm{D}_{3} \mathrm{X}_{\mathrm{m}} \cdot \mathrm{D}_{1}, \mathrm{D}_{2}$ e $\mathrm{D}_{3}$ são as variáveis "Dummy" para as classes de produtividade alta, média e baixa, respectivamente; $X_{1}, X_{2}, X_{m}$ são variáveis independentes da regressão linear múltipla.

Com o modelo completo, testou-se a hipótese $\mathrm{H}_{0}: \mathrm{b}_{11}=\mathrm{b}_{12}=\mathrm{b}_{\mathrm{sm}}=0$ contra a hipótese alternativa $\mathrm{H}_{1}: \mathrm{b}_{11} \neq \mathrm{b}_{12} \neq \mathrm{b}_{\mathrm{sm}} \neq 0$. A hipótese $\mathrm{H}_{0}$ assume um único modelo para todas as classes de produtividade, chamado de modelo reduzido $\left(\mathrm{M}_{\mathrm{r}}\right)$, que tem a seguinte estrutura: $M_{r}: \hat{Y}=b_{1} X_{1}+b_{2} X_{2}+b_{3} X_{3}+b_{4} X_{4}$, em que $b_{1}, b_{2}, b_{3}, b_{4}$ são os coeficientes da regressão linear múltipla; e $\mathrm{X}_{1}, \mathrm{X}_{2}, \mathrm{X}_{3}, \mathrm{X}_{4}$ são as variáveis independentes da regressão linear múltipla.

A verificação da hipótese $\mathrm{H}_{0}$ foi realizada com o teste $\mathrm{F}$ da ANOVA para a redução (Neter et al., 1996; Schneider \& Tonini, 2003), a 5\% de probabilidade.

\section{Resultados e Discussão}

O comportamento dos índices de competição foi verificado pelo agrupamento dos dados das árvores em três classes de tamanho: maiores, com DAP > $\overline{\mathrm{x}}+0,5 \mathrm{~s} ;$ médias, com $\overline{\mathrm{x}}+0,5 \mathrm{~s} \leq \mathrm{DAP} \leq \overline{\mathrm{x}}+0,5 \mathrm{~s}$; e menores, com DAP $<\overline{\mathrm{x}}-0,5 \mathrm{~s}$; para as três classes de produtividade (alta: $\overline{\mathrm{x}}=15,5$ e $\mathrm{s}=7$; média: $\overline{\mathrm{x}}=14$ e $\mathrm{s}=6$; e baixa: $\overline{\mathrm{x}}=13$ e $\mathrm{s}=6$ ) (Figura 1).

Os índices $\mathrm{IID}_{1}, \mathrm{IID}_{2}, \mathrm{IID}_{3}$ e $\mathrm{IID}_{4}$ mostraram tendência crescente para as árvores maiores, tendência constante (valor próximo de 1,0) para árvores médias e tendência decrescente para as árvores menores (Figura 1). Esses índices apresentam variáveis do povoamento no denominador (altura média, diâmetro médio), que aumentam com a idade do povoamento, o que indica que as árvores menores têm menor vigor de crescimento e as árvores maiores apresentam taxas maiores de crescimento.

O índice de competição IID5, que é o somatório das áreas seccionais das árvores maiores do que a árvore-objeto, apresentou tendência crescente para as três classes de tamanho. Além disso, contrariamente ao que ocorre com os índices anteriores, árvores menores apresentam valores maiores para o $\mathrm{IID}_{5}$, em razão da forma de cálculo do índice.

As correlações entre os índices de competição e o crescimento dimensional em diâmetro variaram entre $-0,74$ e 0,52 para as classes de produtividade baixa e alta, respectivamente (Tabela 2). Os índices $\mathrm{IID}_{4}$ e $\mathrm{IID}_{5}$ tiveram maiores valores, respectivamente: 0,70 e $-0,74$, na classe de produtividade alta; 0,64 e -0,64, na classe de produtividade média; e 0,62 e $-0,63$, na classe de produtividade baixa. Esses valores foram ligeiramente superiores aos encontrados por: Monserud \& Sterba (1999), que encontraram correlações entre -0,33 a -0,50, para algumas espécies na Áustria; Sánchez-González et al. (2006), que encontraram valores de $-0,34$ entre o $\mathrm{IID}_{5}$ e o crescimento em diâmetro de Quercus suber L.; Davies \& Pommerening (2008), que encontraram 
valores de $-0,42$, para Betula spp., e -0,75, para Picea sitchensis (Bong.) Carrière, nas florestas da Escócia.

Todos os índices de competição, exceto o $\mathrm{IID}_{5}$, apresentaram correlações positivas quanto ao crescimento em diâmetro e altura. Para a probabilidade de mortalidade, todas as correlações foram negativas, ou seja, quanto maior o valor do índice de competição, maior a capacidade em competir com as árvores vizinhas e menor a probabilidade de morrer.

Trabalhos que avaliaram diferentes índices de competição independentes da distância também obtiveram valores menores que os encontrados no
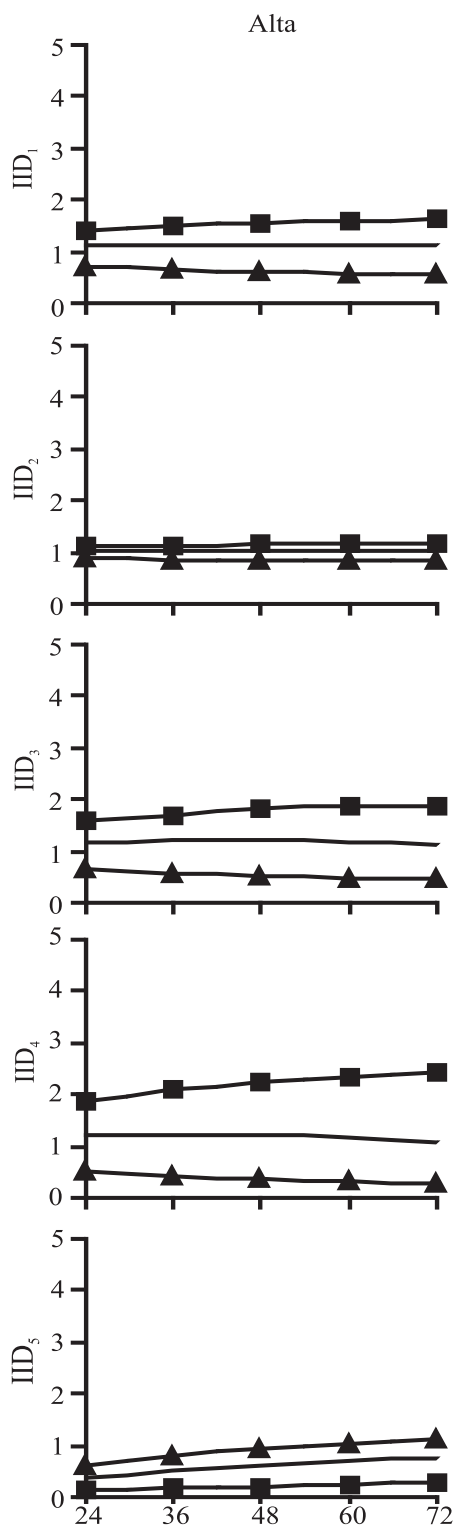
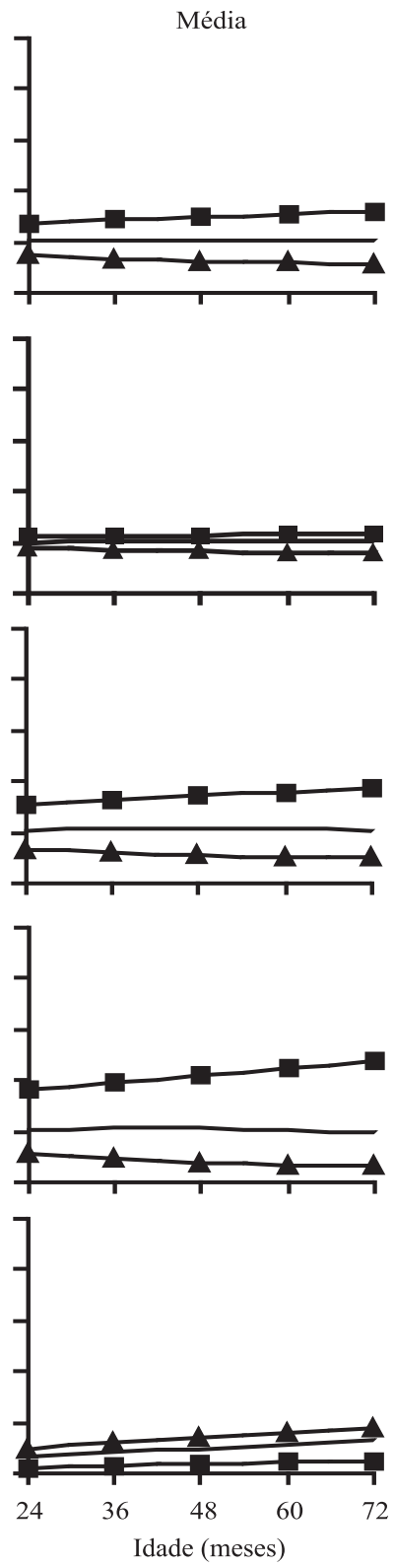

Maiores

Médias

Menores
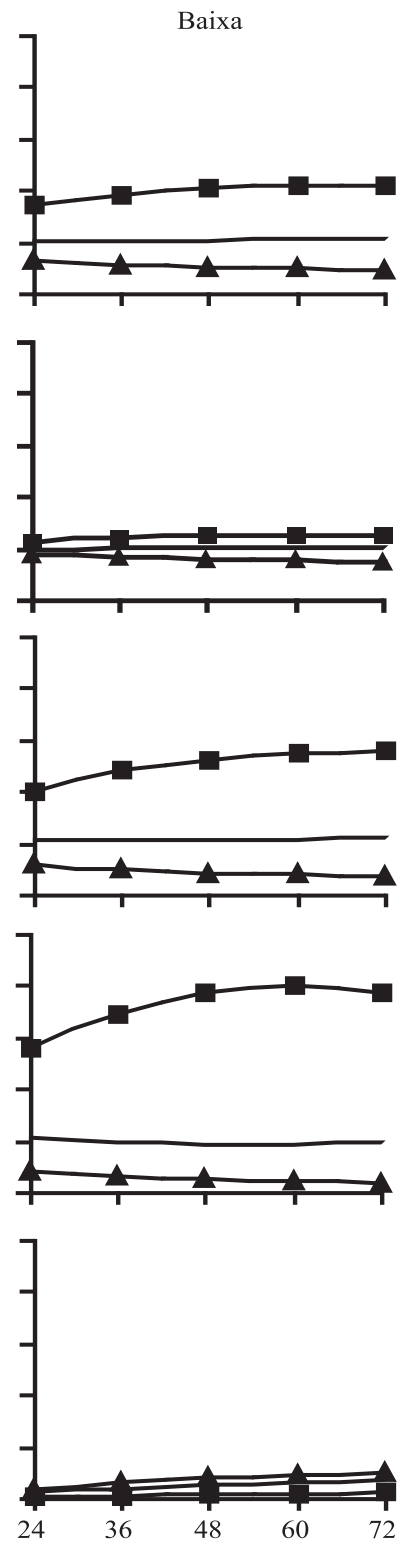

Figura 1. Comportamento médio dos índices de competição independentes da distância $\left(\operatorname{IID}_{1}\right.$ à $\left.\operatorname{IID}_{5}\right)$, em função da idade de árvores individuais de Eucalyptus grandis $\mathrm{x}$ Eucalyptus urophylla, nas classes de tamanho maiores (DAP $>\overline{\mathrm{x}}+0,5 \mathrm{~s})$, médias $(\overline{\mathrm{x}}-0,5 \mathrm{~s} \leq \mathrm{DAP} \leq+0,5 \mathrm{~s})$ e menores (DAP $<\overline{\mathrm{x}}-0,5 \mathrm{~s})$ e nas classes de produtividade alta $(\mathrm{S}=$ 32): altura média das árvores dominantes $(\overline{\mathrm{Hd}})>29 \mathrm{~m}$; classe de produtividade média $(\mathrm{S}=26)$ : $\overline{\mathrm{Hd}}$ entre 24 e $29 \mathrm{~m}$; classe de produtividade baixa $(\mathrm{S}=20): \overline{\mathrm{Hd}} \leq 23 \mathrm{~m}$. 
presente trabalho. Como exemplo, tem-se o índice de Lorimer, que considera o somatório da razão entre diâmetros das árvores competidoras e a árvore-objeto, com valores iguais a $-0,48$ e - 0,55 para Acer rubrum $\mathrm{L}$. e Quercus rubra L., respectivamente, e o índice da razão entre o diâmetro da árvore-objeto e a média do diâmetro das suas competidoras, com valores entre 0,23 e 0,31 para Pinus taeda L. (Daniels et al., 1986).

Quanto ao crescimento em diâmetro, as correlações simples (em módulo) de $\mathrm{IID}_{4}$ e $\mathrm{IID}_{5}$ foram bem próximas nas classes de produtividade alta e baixa, e foram iguais na classe de produtividade média (Tabela 2). Além disso, os valores foram inferiores na menor classe de produtividade, o que mostrou que o índice de competição foi mais importante, quando calculado em classes de maior produtividade.

Weiner \& Thomas (1986) mostraram a relação entre competição e índice de local, e concluíram que a competição é diretamente proporcional ao índice de local, e que as plantas localizadas em um local de maior capacidade produtiva são mais aptas a competir pelos mesmos recursos do que plantas em um local de menor capacidade produtiva. Além disso, plantas em locais de maior produtividade crescem e competem, enquanto a sobrevivência é prioridade em locais de menor produtividade.

Tabela 2. Matriz de correlação simples entre os índices de competição independentes da distância $\left(\operatorname{IID}_{1}\right.$ a $\left.\operatorname{IID}_{5}\right)$, crescimento dimensional em diâmetro $(\Delta \mathrm{d})$ e altura $(\Delta \mathrm{h})$, probabilidade de mortalidade $(\mathrm{PM})$, idade (I) e área basal (B), nas classes de produtividade $^{(1)}$ de árvores individuais de Eucalyptus grandis x Eucalyptus urophylla.

\begin{tabular}{|c|c|c|c|c|c|c|c|c|c|c|}
\hline Parâmetro & $\mathrm{I}$ & $\mathrm{IID}_{1}$ & $\mathrm{IID}_{2}$ & $\mathrm{IID}_{3}$ & $\mathrm{IID}_{4}$ & $\mathrm{IID}_{5}$ & $\Delta \mathrm{d}$ & $\Delta \mathrm{h}$ & PM & $\mathrm{B}$ \\
\hline & \multicolumn{10}{|c|}{ Produtividade alta } \\
\hline I & 1,00 & $-0,05$ & $-0,07$ & $-0,04$ & $-0,06$ & $-0,32$ & $-0,34$ & $-0,74$ & $-0,57$ & 0,89 \\
\hline $\mathrm{IID}_{1}$ & - & 1,00 & 0,97 & 1,00 & 0,99 & $-0,92$ & 0,68 & 0,44 & $-0,54$ & $-0,06$ \\
\hline $\mathrm{IID}_{2}$ & - & - & 1,00 & 0,95 & 0,97 & $-0,92$ & 0,65 & 0,47 & $-0,57$ & $-0,07$ \\
\hline $\mathrm{IID}_{3}$ & - & - & - & 1,00 & 0,99 & $-0,88$ & 0,67 & 0,43 & $-0,43$ & $-0,05$ \\
\hline $\mathrm{IID}_{4}$ & - & - & - & - & 1,00 & $-0,91$ & 0,70 & 0,55 & $-0,62$ & $-0,06$ \\
\hline $\mathrm{IID}_{5}$ & - & - & - & - & - & 1,00 & $-0,74$ & $-0,60$ & $-0,59$ & $-0,34$ \\
\hline$\Delta \mathrm{d}$ & - & - & - & - & - & - & 1,00 & 0,82 & $-0,71$ & $-0,36$ \\
\hline$\Delta \mathrm{h}$ & - & - & - & - & - & - & - & 1,00 & $-0,45$ & $-0,68$ \\
\hline PM & - & - & - & - & - & - & - & - & 1,00 & $-0,13$ \\
\hline \multirow[t]{2}{*}{$\mathrm{B}$} & - & - & - & - & - & - & - & - & - & 1,00 \\
\hline & \multicolumn{10}{|c|}{ Produtividade média } \\
\hline I & 1,00 & 0,01 & $-0,01$ & 0,02 & $-0,01$ & 0,46 & $-0,36$ & $-0,68$ & $-0,31$ & 0,79 \\
\hline $\mathrm{IID}_{1}$ & - & 1,00 & 0,97 & 0,98 & 0,98 & $-0,76$ & 0,63 & 0,41 & $-0,38$ & $-0,02$ \\
\hline $\mathrm{IID}_{2}$ & - & - & 1,00 & 0,95 & 0,98 & $-0,76$ & 0,62 & 0,45 & $-0,38$ & $-0,02$ \\
\hline $\mathrm{IID}_{3}$ & - & - & - & 1,00 & 0,99 & $-0,75$ & 0,62 & 0,39 & $-0,35$ & $-0,01$ \\
\hline $\mathrm{IID}_{4}$ & - & - & - & - & 1,00 & $-0,78$ & 0,64 & 0,52 & $-0,47$ & $-0,02$ \\
\hline $\mathrm{IID}_{5}$ & - & - & - & - & - & 1,00 & $-0,64$ & $-0,59$ & $-0,39$ & $-0,02$ \\
\hline$\Delta \mathrm{d}$ & - & - & - & - & - & - & 1,00 & 0,87 & $-0,43$ & $-0,24$ \\
\hline$\Delta \mathrm{h}$ & - & - & - & - & - & - & - & 1,00 & $-0,40$ & $-0,48$ \\
\hline PM & - & - & - & - & - & - & - & - & 1,00 & $-0,22$ \\
\hline \multirow[t]{2}{*}{ B } & - & - & - & - & - & - & - & - & - & 1,00 \\
\hline & \multicolumn{10}{|c|}{ Produtividade baixa } \\
\hline I & 1,00 & 0,01 & $-0,02$ & 0,02 & $-0,02$ & 0,42 & $-0,33$ & $-0,49$ & $-0,30$ & 0,90 \\
\hline $\mathrm{IID}_{1}$ & - & 1,00 & 0,98 & 0,99 & 0,99 & $-0,84$ & 0,52 & 0,58 & $-0,30$ & $-0,01$ \\
\hline $\mathrm{IID}_{2}$ & - & - & 1,00 & 0,96 & 0,98 & $-0,86$ & 0,54 & 0,57 & $-0,11$ & $-0,01$ \\
\hline $\mathrm{IID}_{3}$ & - & - & - & 1,00 & 0,99 & $-0,82$ & 0,55 & 0,54 & $-0,28$ & $-0,02$ \\
\hline $\mathrm{IID}_{4}$ & - & - & - & - & 1,00 & $-0,86$ & 0,62 & 0,58 & $-0,50$ & $-0,02$ \\
\hline $\mathrm{IID}_{5}$ & - & - & - & - & - & 1,00 & $-0,63$ & $-0,69$ & $-0,32$ & $-0,46$ \\
\hline$\Delta \mathrm{d}$ & - & - & - & - & - & - & 1,00 & 0,94 & $-0,53$ & $-0,35$ \\
\hline$\Delta \mathrm{h}$ & - & - & - & - & - & - & - & 1,00 & $-0,46$ & $-0,47$ \\
\hline PM & - & - & - & - & - & - & - & - & 1,00 & $-0,46$ \\
\hline B & - & - & - & - & - & - & - & - & - & 1,00 \\
\hline
\end{tabular}

${ }^{(1)}$ Classe de produtividade alta (índice de local, $\mathrm{S}=32$ ): altura média das árvores dominantes $(\overline{\mathrm{Hd}})>29 \mathrm{~m}$; classe de produtividade média $(\mathrm{S}=26)$ : $\overline{\mathrm{Hd}}$ entre 24 e $29 \mathrm{~m}$; classe de produtividade baixa $(\mathrm{S}=20): \overline{\mathrm{Hd}} \leq 23 \mathrm{~m}$. 
Nas correlações simples entre os índices de competição e o crescimento dimensional em altura, verificou-se que os índices IID $_{4}$ e IID $_{5}$ também apresentaram leve superioridade em relação aos outros índices avaliados, com valores de 0,52 a -0,69. Os valores apresentados no presente trabalho também foram maiores do que outros encontrados na literatura quanto ao crescimento em altura. Martin \& Ek (1984) encontraram valor de 0,38 para o crescimento em altura de Pinus resinosa Aiton, e Chassot et al. (2011) valores inferiores a -0,64 para Araucaria angustifolia (Bertol.) Kuntze.

Em comparação aos índices dependentes da distância, as correlações encontradas no presente trabalho foram ligeiramente superiores aos encontrados na literatura. Valores inferiores a $-0,41$ foram encontrados para crescimento em diâmetro e $-0,45$ para crescimento em altura, com o uso de diferentes índices dependentes da distância; os inferiores a $-0,53$ foram encontrados pelo índice de Hegyi; inferiores a $-0,36$, com o índice Bella; não maiores que $-0,55$, com o conceito de área potencialmente disponível; inferiores a $-0,63$, com índices que utilizam o conceito de área sobreposta (Daniels, 1976; Daniels et al., 1986; Tomé \& Burkhart, 1989; Sánchez-González et al., 2006; Filipescu \& Comeau, 2007; Fox et al., 2007; Weber et al., 2008; Martins, 2011).

Por meio das correlações simples entre os índices de competição e a PM, verificou-se que $\mathrm{IID}_{4}$ e $\mathrm{IID}_{5}$ apresentaram, mais uma vez, leve superioridade em relação aos demais índices (Tabela 2). Os índices $\mathrm{IID}_{4}$ e $\mathrm{IID}_{5}$ apresentaram correlações maiores para o local de maior produtividade e correlações menores para os locais de produtividade média e baixa. Esses resultados mostram que os índices de competição são importantes no cálculo da probabilidade de mortalidade, principalmente nos locais onde a competição por recursos é maior, semelhantemente às tendências observadas no crescimento em diâmetro e altura.

A correlação simples com o crescimento em diâmetro e altura tem sido um critério comum para a seleção de índices de competição (Weber et al., 2008). No presente trabalho, os índices mais correlacionados ao crescimento em diâmetro e altura e à PM foram o IID $_{4}$ e o IID $_{5}$. No entanto, é ainda pouco estudado o comportamento dos índices de competição, em modelos de regressão utilizados para projetar o crescimento e a produção de povoamentos florestais,
(Sánchez-González et al., 2006; Filipescu \& Comeau, 2007). Assim, foram ajustadas equações lineares múltiplas, tendo-se considerado como variáveis dependentes o crescimento em diâmetro e altura e a probabilidade de mortalidade, em função de variáveis do povoamento idade e área basal, DAP e índice de competição (Tabela 3).

As equações se ajustaram bem aos dados observados de crescimento em diâmetro e em altura, com $\overline{\mathrm{R}}^{2}$ maior que $90 \%$, considerando-se o $\mathrm{IID}_{4}$. As equações para o crescimento em altura também se ajustaram bem aos dados observados para o IID $_{5}$, com $\overline{\mathrm{R}}^{2}$ maior que $99 \%$. As equações da probabilidade de mortalidade apresentaram $\overline{\mathrm{R}}^{2}$ menores do que os das equações de crescimento em diâmetro e em altura $\left(\overline{\mathrm{R}}^{2}\right.$ entre $0,1394 \mathrm{e}$ $0,5761)$. Valores inferiores para as medidas de precisão foram encontrados em trabalhos de Martin \& Ek (1984), Daniels et al. (1986) e Sánchez-González et al. (2006) para o crescimento em diâmetro e altura de $P$. resinosa, $P$. taeda e $Q$. suber, respectivamente. No entanto, Schneider et al. (2005) encontraram valores de precisão superiores aos verificados no presente trabalho para a probabilidade de mortalidade.

Com relação ao crescimento em diâmetro e em altura, os parâmetros estatísticos foram melhores para os locais de média produtividade, seguidos pelos locais de alta e baixa produtividade. Para a probabilidade de mortalidade, os valores de $\overline{\mathrm{R}}^{2}$ e $\mathrm{S}_{\mathrm{yx}} \%$ foram superiores para os locais de alta produtividade e inferiores para os locais de média produtividade.

Os coeficientes associados às variáveis explicativas, nas equações de crescimento em diâmetro e em altura $\left(\beta_{1}, \beta_{2}\right.$ e $\left.\beta_{3}\right)$, foram significativos $(p<0,05)$, em todas as classes de produtividade, independentemente do índice de competição. Os coeficientes associados ao $\mathrm{IID}_{4}$, nas equações da probabilidade de mortalidade, foram significativos independentemente da classe de produtividade. Contudo, os coeficientes associados ao $\mathrm{IID}_{5}$ não foram significativos.

A contribuição do IID $_{4}$ foi significativa para o crescimento em diâmetro e altura, e também para a probabilidade de mortalidade, independentemente da classe de produtividade (Tabela 3 ). $\mathrm{O}_{\mathrm{IID}_{5}}$ não foi significativo para: crescimento em diâmetro na classe de produtividade baixa; crescimento em altura na classe de produtividade média; e probabilidade de mortalidade nas três classes de produtividade. Além disso, o $\mathrm{IID}_{4}$ apresentou menor contribuição para 
a probabilidade de mortalidade em comparação ao crescimento em diâmetro e altura.

Com exceção da probabilidade de mortalidade para as classes de produtividade média e baixa, o valor de F-parcial para o IID $_{4}$ diminuiu do local de maior produtividade para o de menor produtividade, o que mostra que quanto melhor o local de plantio, maior o efeito da competição no crescimento das árvores. Esse resultado corroborou o índice de competição como uma boa variável explicativa para as melhores classes de produtividade (Fox et al., 2007).

$\mathrm{O} I I D_{4}$ foi utilizado com sucesso por Tomé \& Burkhart (1989), Martins (2011) e outros. O IID $_{5}$ foi utilizado nos modelos de árvore individual Prognosis (Davis et al., 2005), Prognaus (Sterba \& Monserud, 1997) e TWIGS (Monserud \& Sterba, 1999). O IID $_{5}$ também foi utilizado com sucesso em estudos com espécies de coníferas, em povoamentos mistos e tropicais, em florestas da Áustria e da Espanha (Sánchez-González et al., 2006).
Monserud \& Sterba (1999) e Eid \& Tuhus (2001) salientaram que o $\operatorname{IID}_{5}$ é um excelente índice de competição, pois não necessita de informações espaciais de cada árvore; no entanto, deve ser associado a uma medida da copa das árvores (fator de copa), para se avaliar melhor a competição. $\mathrm{O} \mathrm{IID}_{4}$ também não necessita de informações espaciais e, além disso, não precisa do fator de copa, pois já contem associadas em sua fórmula as variáveis do povoamento e do tamanho da árvore. Assim, o $\mathrm{IID}_{4}$ pode ser considerado um índice mais simples do que o IID $_{5}$, além de apresentar significado biológico e um bom desempenho na avaliação da competição.

Após avaliar o desempenho dos índices de competição e selecionar o $\mathrm{IID}_{4}$ como melhor índice de competição, para plantios clonais híbridos não desbastados de Eucalyptus grandis x Eucalyptus urophylla, foi realizado o teste de identidade de modelos com a variável "Dummy", para o crescimento em diâmetro e altura e a probabilidade de mortalidade.

Tabela 3. Coeficientes e parâmetros estatísticos e teste $\mathrm{F}$ parcial das regressões múltiplas, para estimar o crescimento em diâmetro e altura e a probabilidade de mortalidade, nas classes de produtividade com os índices de competição independentes da distância $\mathrm{IID}_{4}$ e $\mathrm{IID}_{5}$ de árvores individuais de Eucalyptus grandis x Eucalyptus urophylla ${ }^{(1)}$.

\begin{tabular}{|c|c|c|c|c|c|c|c|c|}
\hline \multirow[t]{2}{*}{ Classe de produtividade ${ }^{(1)}$} & \multirow[t]{2}{*}{ IC } & \multicolumn{4}{|c|}{ Coeficiente } & \multicolumn{2}{|c|}{ Parâmetro estatístico } & \multirow[t]{2}{*}{ F-parcial ${ }^{(2)}$} \\
\hline & & $\hat{\beta}_{0}$ & $\widehat{\beta_{1}}$ & $\hat{\beta}_{2}$ & $\hat{\beta}_{3}$ & $\overline{\mathrm{R}}^{2}(\%)$ & $\mathrm{S}_{\mathrm{yx}}(\%)$ & \\
\hline & \multicolumn{8}{|c|}{ Crescimento em diâmetro } \\
\hline \multirow[t]{2}{*}{ Alta } & $\mathrm{IID}_{4}$ & $-0,3169$ & 0,0596 & 7,6778 & 0,2539 & 0,9524 & $\pm 5,46$ & $209,4224 *$ \\
\hline & $\mathrm{IID}_{5}$ & 14,0142 & 0,1737 & $-11,9526$ & - & 0,8577 & $\pm 9,44$ & $187,4497 *$ \\
\hline \multirow[t]{2}{*}{ Média } & $\mathrm{IID}_{4}$ & $-0,2312$ & 0,0328 & 6,8421 & 0,3427 & 0,9616 & $\pm 5,64$ & $159,3996^{*}$ \\
\hline & $\mathrm{IID}_{5}$ & 9,4361 & 0,1892 & $-10,8480$ & - & 0,6458 & $\pm 12,14$ & $28,3481^{*}$ \\
\hline \multirow[t]{3}{*}{ Baixa } & $\mathrm{IID}_{4}$ & $-0,5367$ & 0,0176 & 5,2033 & 0,0214 & 0,9193 & $\pm 8,24$ & $4,4761 *$ \\
\hline & $\mathrm{IID}_{5}$ & 8,6151 & 0,1580 & $-18,0311$ & - & 0,8046 & $\pm 10,81$ & $0,0798^{\text {ns }}$ \\
\hline & \multicolumn{8}{|c|}{ Crescimento em altura } \\
\hline \multirow[t]{2}{*}{ Alta } & $\mathrm{IID}_{4}$ & $-0,1224$ & 0,1674 & 7,2674 & 0,3705 & 0,9427 & $\pm 5,90$ & $129,2693 *$ \\
\hline & $\mathrm{IID}_{5}$ & $-1,1296$ & 0,1015 & 1,1342 & 2,3883 & 0,9924 & $\pm 1,85$ & $19,1893^{*}$ \\
\hline \multirow[t]{2}{*}{ Média } & $\mathrm{IID}_{4}$ & 0,8181 & 0,1278 & 6,5897 & 0,4189 & 0,9559 & $\pm 5,29$ & $109,0264 *$ \\
\hline & $\mathrm{IID}_{5}$ & 0,2625 & 0,0911 & 1,0820 & 1,6533 & 0,9915 & $\pm 2,31$ & $1,4825^{\mathrm{ns}}$ \\
\hline \multirow[t]{2}{*}{ Baixa } & $\mathrm{IID}_{4}$ & 0,5446 & 0,0929 & 5,1515 & 0,6519 & 0,9234 & $\pm 6,65$ & $17,3345^{*}$ \\
\hline & $\mathrm{IID}_{5}$ & 0,7250 & 0,0758 & 1,0829 & 1,6042 & 0,9931 & $\pm 1,99$ & $7,6233^{*}$ \\
\hline & \multicolumn{8}{|c|}{ Probabilidade de mortalidade } \\
\hline \multirow[t]{2}{*}{ Alta } & $\mathrm{IID}_{4}$ & 0,3789 & $-0,0038$ & $-0,1867$ & - & 0,5513 & $\pm 17,26$ & $55,9536^{*}$ \\
\hline & $\mathrm{IID}_{5}$ & 0,4742 & $-0,0002^{\mathrm{ns}}$ & $-0,0210$ & $-0,0816^{\mathrm{ns}}$ & 0,5761 & $\pm 17,67$ & $1,5389^{\mathrm{ns}}$ \\
\hline \multirow[t]{2}{*}{ Média } & $\mathrm{IID}_{4}$ & 0,1288 & $-0,0006^{\mathrm{ns}}$ & $-0,0679$ & - & 0,1394 & $\pm 27,48$ & $6,5762 *$ \\
\hline & $\mathrm{IID}_{5}$ & 0,1522 & $-0,0008^{\mathrm{ns}}$ & $-0,0097$ & $-0,0599^{\mathrm{ns}}$ & 0,1421 & $\pm 27,43$ & $0,9499^{\text {ns }}$ \\
\hline \multirow[t]{2}{*}{ Baixa } & $\mathrm{IID}_{4}$ & 0,2378 & $-0,0024$ & $-0,1117$ & - & 0,4365 & $\pm 26,82$ & $15,5525^{*}$ \\
\hline & $\mathrm{IID}_{5}$ & 0,2207 & $-0,0020$ & $-0,0127$ & $-0,0873^{\mathrm{ns}}$ & 0,3998 & $\pm 25,47$ & $0,4380^{\text {ns }}$ \\
\hline
\end{tabular}

${ }^{(1)}$ Classe de produtividade alta (índice de local, $\mathrm{S}=32$ ): altura média das árvores dominantes $(\overline{\mathrm{Hd}})>29 \mathrm{~m}$; classe de produtividade média $(\mathrm{S}=26)$ : $\overline{\mathrm{Hd}}$ entre 24 e $29 \mathrm{~m}$; classe de produtividade baixa $(\mathrm{S}=20): \overline{\mathrm{Hd}} \leq 23 \mathrm{~m}$. Modelos teóricos utilizados no ajuste das equações de crescimento em diâmetro, com o índice $\mathrm{IID}_{4}(\mathrm{BAI}): \Delta \mathrm{d}=\mathrm{f}\left(\mathrm{I}_{,} \mathrm{IID}_{4}, \mathrm{~B}\right)$; com o índice $\mathrm{IID}_{5}(\mathrm{BAL}): \Delta \mathrm{d}=\mathrm{f}\left(\mathrm{I}, \mathrm{IID}_{5}\right)$; crescimento em altura, com o índice $\mathrm{IID}_{4}(\mathrm{BAI}): \Delta \mathrm{h}=\mathrm{f}\left(\mathrm{I}, \mathrm{IID}_{4}, \mathrm{~B}\right) ; \mathrm{com}_{\mathrm{o}}$ $\mathrm{IID}_{5}(\mathrm{BAL}): \Delta \mathrm{h}=\mathrm{f}\left(\mathrm{I}, \mathrm{DAP}\right.$, IID5); probabilidade de mortalidade, com o índice $\mathrm{IID}_{4}(\mathrm{BAI}): \mathrm{P}(\mathrm{M})=\mathrm{f}\left(\mathrm{I}_{1} \mathrm{IID}_{4}\right)$; com o IID $5(\mathrm{BAL}): \mathrm{P}(\mathrm{M})=\mathrm{f}(\mathrm{I}, \mathrm{DAP}$, IID 5$)$.

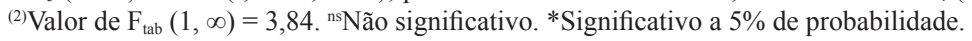


De acordo com os resultados da ANOVA (Tabela 4), houve necessidade de se ajustar uma equação para cada classe de produtividade quanto ao crescimento em diâmetro e ao crescimento em altura. Com relação à probabilidade de mortalidade, foi aceita a hipótese $\mathrm{H}_{0}$ para a redução, o que possibilitou o ajuste de uma única equação para a probabilidade de mortalidade para as três classes de produtividade $\left(\mathrm{F}_{\mathrm{cal}}<\mathrm{F}_{\mathrm{tab}}\right)$.

Rossi et al. (2007) afirmaram que árvores de diferentes espécies morrem, em razão da influência direta da sua capacidade de competir com outras em uma idade específica, independentemente do local em que elas estejam plantadas. Isso justifica o uso, na maioria das vezes, do índice de competição nos modelos de mortalidade para árvore individual (Schneider et al., 2005; Crecente-Campo et al., 2009).

No entanto, Monserud \& Sterba (1999) e Eid \& Tuhus (2001) relacionaram, além do índice de competição, as variáveis de tamanho. Outras variáveis utilizadas para descrever a mortalidade foram: tamanho das árvores e vigor de crescimento e combinação entre tamanho das árvores e variáveis de povoamento (Schneider et al., 2005). Com isso, reforça-se a ideia da relação funcional para descrever a probabilidade de mortalidade, apresentada por Davis et al. (2005),

Tabela 4. Análise de variância do modelo completo e reduzido para crescimento em diâmetro, altura e probabilidade de mortalidade de árvores individuais de Eucalyptus grandis $\mathrm{x}$ Eucalyptus urophylla.

\begin{tabular}{|c|c|c|c|c|c|}
\hline $\mathrm{FV}^{(1)}$ & GL & SQ & QM & $\mathrm{F}_{\mathrm{cal}}$ & $\mathrm{F}_{\mathrm{tab}}$ \\
\hline & \multicolumn{5}{|c|}{ Crescimento em diâmetro } \\
\hline $\operatorname{Par}\left(\mathrm{M}_{\mathrm{c}}\right)$ & 12 & $7.287,320$ & 0,0016 & - & - \\
\hline $\operatorname{Par}\left(\mathrm{M}_{\mathrm{r}}\right)$ & 6 & $7.211,803$ & $1.201,967$ & - & - \\
\hline Redução $\mathrm{H}_{0}$ & 6 & 75,517 & 12,586 & 53,084 & 2,100 \\
\hline Resíduo & 5.941 & $1.408,566$ & 0,2371 & - & - \\
\hline \multirow[t]{2}{*}{ Total } & 5.965 & $8.695,885$ & - & - & - \\
\hline & \multicolumn{5}{|c|}{ Crescimento em altura } \\
\hline $\operatorname{Par}\left(\mathrm{M}_{\mathrm{c}}\right)$ & 12 & $31.219,91$ & $2.601,66$ & - & - \\
\hline $\operatorname{Par}\left(\mathrm{M}_{\mathrm{r}}\right)$ & 6 & $31.001,36$ & $5.166,89$ & - & - \\
\hline Redução $\mathrm{H}_{0}$ & 6 & 218,55 & 36,42 & 106,38 & 2,100 \\
\hline Resíduo & 5.941 & $2.034,23$ & 0,34 & - & - \\
\hline \multirow[t]{2}{*}{ Total } & 5.965 & $33.254,14$ & - & - & - \\
\hline & \multicolumn{5}{|c|}{ Probabilidade de mortalidade } \\
\hline $\operatorname{Par}\left(\mathrm{M}_{\mathrm{c}}\right)$ & 9 & 3,0205 & 0,3356 & - & - \\
\hline $\operatorname{Par}\left(\mathrm{M}_{\mathrm{r}}\right)$ & 5 & 2,9884 & 0,5976 & - & - \\
\hline Redução $\mathrm{H}_{0}$ & 4 & 0,0320 & 0,0080 & 2,2870 & 2,4196 \\
\hline Resíduo & 188 & 0,6591 & 0,0035 & - & - \\
\hline Total & 206 & 3,6796 & - & - & - \\
\hline
\end{tabular}

em que esta variável depende das variáveis índice de competição, tamanho da árvore e variáveis de povoamento, as quais são biologicamente conectadas ao processo de mortalidade em um modelo de árvore individual (Crecente-Campo et al., 2009).

\section{Conclusões}

1. $\mathrm{O} \mathrm{IID}_{4}$ é o melhor índice para descrever a competição entre árvores, em plantios comerciais clonais híbridos não desbastados de Eucalyptus grandis $\mathrm{x}$ Eucalyptus urophylla.

2. A superioridade do $\operatorname{IID}_{4}$ deve-se à simplicidade, facilidade de cálculo e seu realismo biológico, uma vez que combina variáveis de tamanho da árvore e de densidade do povoamento.

3. Não há possibilidade de se ajustar uma equação única para o crescimento em diâmetro e altura, para as três classes de produtividade.

4. Há possibilidade de se ajustar uma equação única para a probabilidade de mortalidade, para as três classes de produtividade.

\section{Agradecimentos}

Ao Conselho Nacional de Desenvolvimento Científico e Tecnológico, por concessão de bolsas.

\section{Referências}

CAO, Q.V. A method to distribute mortality in diameter distribution models. Forest Science, v.43, p.435-442, 1997.

CAO, Q.V. Predictions of individual-tree and whole-stand attributes for loblolly pine plantations. Forest Ecology and Management, v.236, p.342-347, 2006.

CASTAGNERI, D.; VACCHIANO, G.; LINGUA, E.; MOTTA, R. Analysis of intraspecific competition in two subalpine Norway spruce (Picea abies (L.) Karst.) stands in Paneveggio (Trento, Italy). Forest Ecology and Management, v.255, p.651-659, 2008.

CHASSOT, T.; FLEIG, F.D.; FINGER, C.A.G.; LONGHI, S.J. Modelos de crescimento em diâmetro de árvores individuais de Araucaria angustifolia (Bertol.) Kuntze na Floresta Ombrófila Mista. Ciência Florestal, v.21, p.303-313, 2011.

CRECENTE-CAMPO, F.; MARSHALL, P.; RODRIGUEZ-SOALLEIRO, R. Modeling non-catastrophic individual tree mortality for Pinus radiata plantations in Northwestern Spain. Forest Ecology and Management, v.257, p.1542-1550, 2009.

CUNHA, T.A. da. Modelagem do incremento de árvores individuais de Cedrela odorata L. na Floresta Amazônica. 2009. 
88p. Dissertação (Mestrado) - Universidade Federal de Santa Maria, Santa Maria.

DANIELS, R.F. Simple competition indices and their correlation with annual loblolly pine tree growth. Forest Science, v.22, p.454-456, 1976.

DANIELS, R.F.; BURKHART, H.E.; CLASON, T.R. A comparison of competition measures for predicting growth of loblolly pine trees. Canadian Journal of Forest Research, v.16, p.1230-1237, 1986.

DAVIES, O.; POMMERENING, A. The contribution of structural indices to the modeling of Sitka spruce (Picea sitchensis) and birch (Betula spp.) crowns. Forest Ecology and Management, v.256, p.68-77, 2008.

DAVIS, L.S.; JONHSON, K.N.; BETTINGER, P.; HOWARD, T.E. Forest management: to sustain ecological, economic, and social values. $4^{\text {th }}$ ed. Illinois: Waveland, 2005. 804p.

DEMOLINARI, R.A. de. Crescimento de povoamentos de eucalipto não desbastados. 2006. 72p. Dissertação (Mestrado) Universidade Federal de Viçosa, Viçosa.

EID, T.; TUHUS, E. Models for individual tree mortality in Norway. Forest Ecology and Management, v.154, p.69-84, 2001.

FILIPESCU, C.N.; COMEAU, P.G. Competitive interactions between aspen and white spruce vary with stand age in boreal mixedwoods. Forest Ecology and Management, v.247, p.175-184, 2007.

FOX, J.C.; BI, H.; ADES, P.K. Spatial dependence and individual-tree growth models. I. Characterising spatial dependence. Forest Ecology and Management, v.245, p.10-19, 2007.

GUJARATI, D.N. Econometria básica. 2.ed. Rio de Janeiro: Elsevier, 2006. 812p.

MARTIN, G.L.; EK, A.R. A comparison of competition measures and growth models for predicting plantation red pine diameter and height growth. Forest Science, v.30, p.731-743, 1984.

MARTINS, F.B. Modelagem de crescimento em nível de árvore individual para plantios comerciais de eucalipto. 2011. 143p. Tese (Doutorado) - Universidade Federal de Viçosa, Viçosa.

MONSERUD, R.A.; STERBA, H. Modeling individual tree mortality for Austrian forest species. Forest Ecology and Management, v.113, p.109-123, 1999.

NETER, J.;KUTNER,M.H.;NACHTSHEIM,C.J.;WASSERMAN, W. Applied linear statistical models. $4^{\text {th }}$ ed. Chicago: Irwin, 1996. 1408 .
REGAZZI, A.J. Teste para verificar a igualdade de parâmetros e a identidade de modelos de regressão não-linear. Revista Ceres, v.50, p.9-26, 2003.

ROSSI, L.M.B.; KOEHLER, H.S.; SANQUETTA, C.R.; ARCE, J.E. Modelagem da mortalidade em florestas naturais. Revista Floresta, v.37, p.275-291, 2007.

SÁNCHEZ-GONZÁLEZ, M.; RÍO, M. del; CAÑELLAS, I.; MONTERO, G. Distance independent tree diameter growth model for cork oak stands. Forest Ecology and Management, v.225, p.262-270, 2006.

SCHNEIDER, P.R.; FORTES, F. de O.; SOUZA, L.H. da S.; LÚCIO, A.D.; FINGER, C.A.G.; SCHNEIDER, P.S.P. Análise da mortalidade de Acacia mearnsii De Wild. Ciência Florestal, v.15, p.137-143, 2005.

SCHNEIDER, P.R.; TONINI, H. Utilização de variáveis dummy em equações de volume para Acacia mearnsii De Wild. Ciência Florestal, v.13, p.121-129, 2003.

STAGE, A.R.; LEDERMANN, T. Effects of competitor spacing in a new class of individual tree indices of competition: semi-distance-independent indices computed for Bitterlich versus fixed-area plots. Canadian Journal of Forest Research, v.38, p.890-898, 2008.

STERBA, H.; MONSERUD, R.A. Applicability of the forest stand growth simulator PROGNAUS for the Austrian part of the Bohemian Massif. Ecological Modelling, v.98, p.23-34, 1997.

TOMÉ, M.; BURKHART, H.E. Distance-dependent competition measures for predicting growth of individual trees. Forest Science, v.35, p.816-831, 1989.

WEBER, P.; BUGMANN, H.; FONTI, P.; RIGLING, A. Using a retrospective dynamic competition index to reconstruct forest succession. Forest Ecology and Management, v.254, p.96-106, 2008.

WEINER, J.; THOMAS, S.C. Size variability and competition in plant monocultures. Oikos, v.47, p.211-222, 1986.

ZHANG, L.J.; PENG, C.H.; DANG, Q.L. Individual-tree basal area growth models for jack pine and black spruce in Northern Ontario. The Forestry Chronicle, v.80, p.366-374, 2004.

ZHAO, D.H.; BORDERS, B.; WILSON, M.; RATHBUN, S.L. Modeling neighborhood effects on the growth and survival of individual trees in a natural temperate species-rich forest. Ecological Modelling, v.196, p.90-102, 2006.

Recebido em 1ํ de junho de 2011 e aprovado em 9 de agosto de 2011 